\title{
Landschaft für die Erholung am Beispiel der Seeuferplanung am Bodensee, Kanton Thurgau
}

\section{Einleitung}

Flüsse und Seen und ihre Ufer zählen zu unseren beliebtesten Erholungsgebieten; sie sind zweifellos selten. Ihre Zugänglichkeit ist an vielen Orten unwiederbringlich eingeschränkt. Mehr und mehr verschwindet die ursprüngliche Ufervegetation. Die Nachfrage nach dem Seeufer als Erholungsraum oder als Bauplatz ist gerade dann besonders groß, wenn es natur-kulturhistorisch wertvolle - oder einfacher gesagt «schöne» - Landschaften sind. Die Bodenseelandschaft zählt dazu.

\section{Die Aufgabenstellung der Seeuferplanung und die Bedeutung des Bodenseeraumes}

(1) Wegen der doppelten Bedeutung des Bodenseegebietes als Kulturlandschaft und Erholungsgebiet beauftragte der Regierungsrat des Kantons Thurgau das Planungsbüro C. Fingerhuth (heute Fingerhuth und Partner) 1970 mit der Erarbeitung eines Erholungs- und Landschaftsschutzplans für das thurgauische Bodensee- und Rheinufer.

(2) Diese Seeuferplanung wurde 1974 mit einem Erholungs- und Landschaftsschutzplan im Maßstab 1:10000 und entsprechenden Berichten abgeschlossen.1)

Dieser Plan wird seitdem als Entwurf eines kantonalen Teilrichtplanes der Landschaft in einem Teilgebiet des Kantons Thurgau angesehen. Er nimmt wesentliche Aspekte eines kantonalen Teilrichtplanes Landschaft für das Ufergebiet vorweg. (Ein kantonales Baugesetz ist erst seit 1977 in Kraft.)

Der Plan wurde mit seinen Zielen und Maßnahmen vom Regierungsrat geprüft und mit dem Vorbehalt zur Kenntnis genommen, daß er, zusammen und abgestimmt mit weiteren Teilrichtplänen, als Bestandteil der kantonalen Richtplanung gelten könne. Er besitzt bis auf weiteres für kantonale Entscheidungen verwaltungsanweisenden Charakter; Entscheide auf kantonaler Ebene sollten auf ihn ausgerichtet werden.

Der Landschaftsplan wurde 1972/1973 der kantonalen Verwaltung zur Stellungnahme vorgelegt. Weiterhin konnten die betroffenen thurgauischen
Gemeinden zu der allgemeinen Zielsetzung, zur heutigen Landschaftssituation und zu den vorgeschlagenen Maßnahmen, sofern sie das jeweilige Gemeindegebiet betreffen, in einem Vernehmlassungsverfahren 1973 Stellung beziehen. Die Änderungsvorschläge beider Vernehmlassungsverfahren, der Gemeinden und der kantonalen Verwaltung, wurden in die vorliegende überarbeitete Fassung einbezogen, so daß diese Planung das Einverständnis des Regierungsrates, der Behörden und mit wenigen Ausnahmen aller betroffenen Gemeinden besitzt.

(3) Der Landschaftsplan war zudem Grundlage zur Ausscheidung der provisorischen Schutzpläne des BMR (Bundesbeschluß über dringliche Maßnahmen auf dem Gebiete der Raumplanung 1972/ 1973). Er ist heute eine wichtige Grundlage für den kommenden kantonalen Teilrichtplan Landschaft des Kantons Thurgau, der aufgrund des kantonalen Baugesetzes von 1977 für das ganze Kantonsgebiet erarbeitet wird.

(4) Im Leitbild des Kantons Thurgau 19783) wird außerdem fast das gesamte Bodensee- und Rheinufer der Seeuferplanung als besonders schützenswerte Landschaft einerseits und Erholungsgebiet von regionaler Bedeutung andererseits dargestellt und die Maßnahme formuliert, daß der Erholungsund Landschaftsschutzplan (Seeuferplanung) in die Nutzungsplanung überführt werden soll.4)

\section{Ergebnisse und Aussagen der Seeuferplanung}

Die Seeuferplanung hat sich als ausgesprochene Teilbereichsplanung im wesentlichen mit den Teilbereichen Erholung und Landschaftsschutz befaßt. Sie kann als Programm oder Leitbild für das Ufergebiet angesehen werden. Die Ziele und Maßnahmen für den Teilbereich Erholung wurden aus der landschaftlichen Eignung für die Erholung und der Tragfähigkeit der Landschaft entwickelt. Da die Bewertung der Erholungseignung ein besonderes

Martin Schwarze, dipl. Ing., Landschafts- und Raumplaner, Fingerhuth und Partner, Büro für Raumplanung AG, Zürich. 


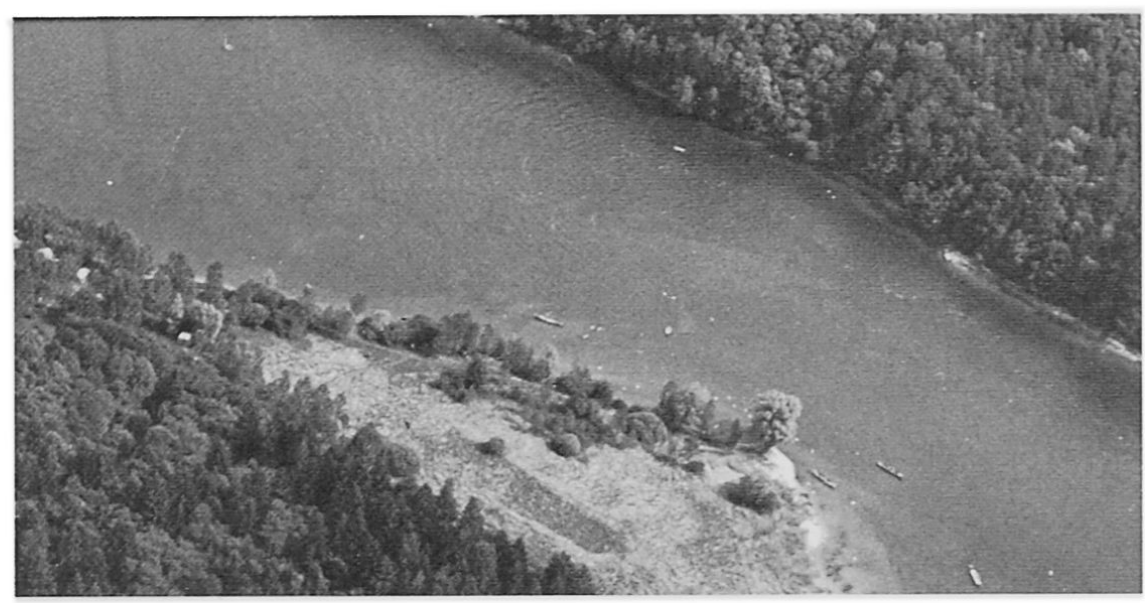

Abb. 1: Wandertyp

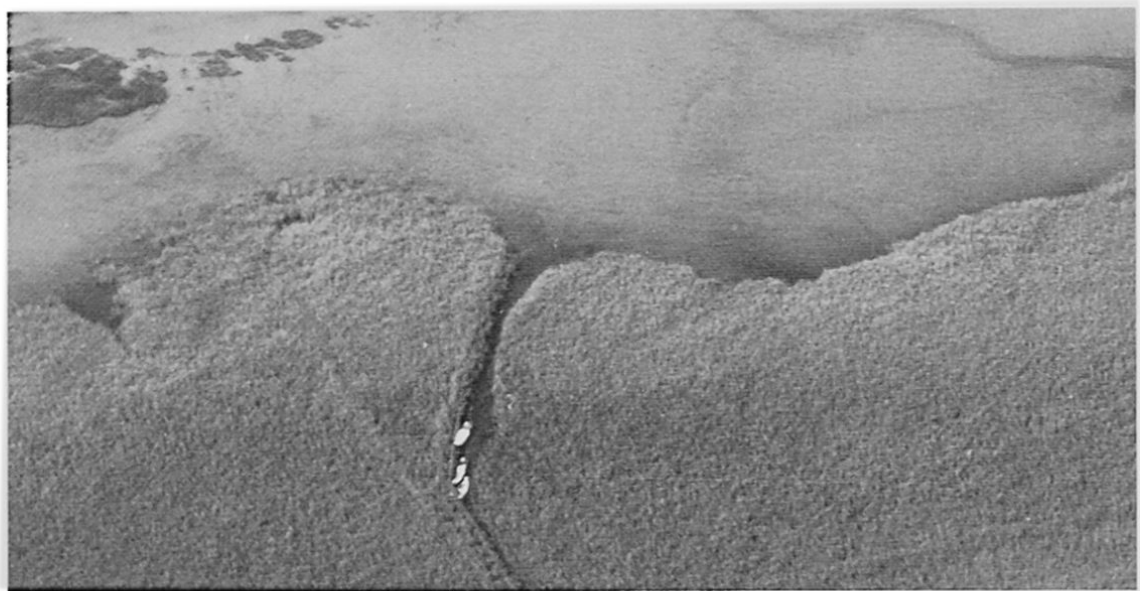

Abb. 2: Freiraumtyp

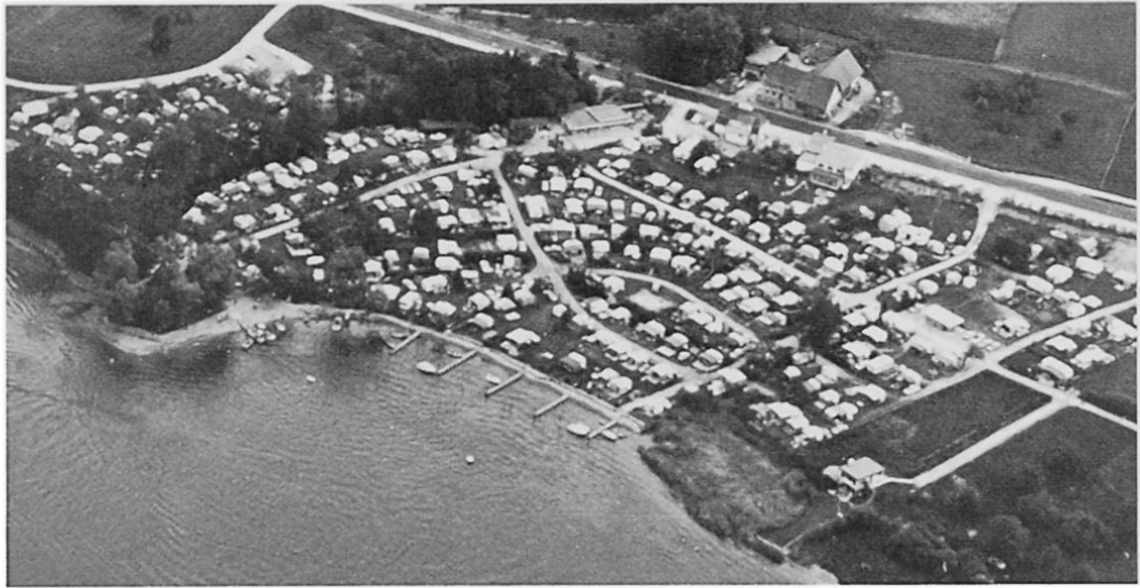

Abb. 3: Lagertyp

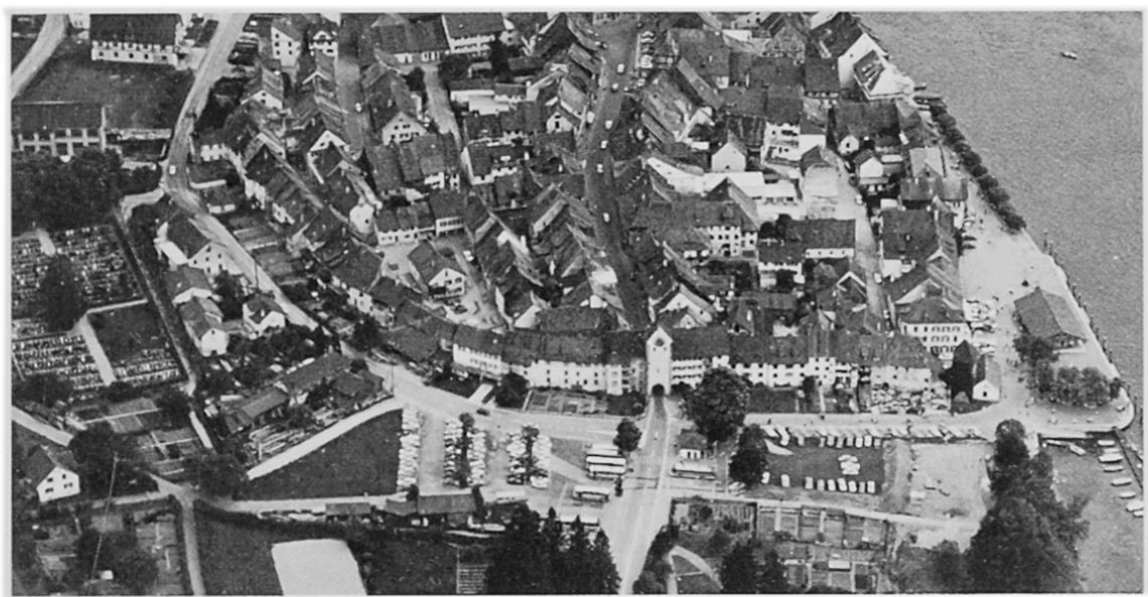

Abb. 4: Rundfahrertyp 
Abb. 5: Promeniertyp

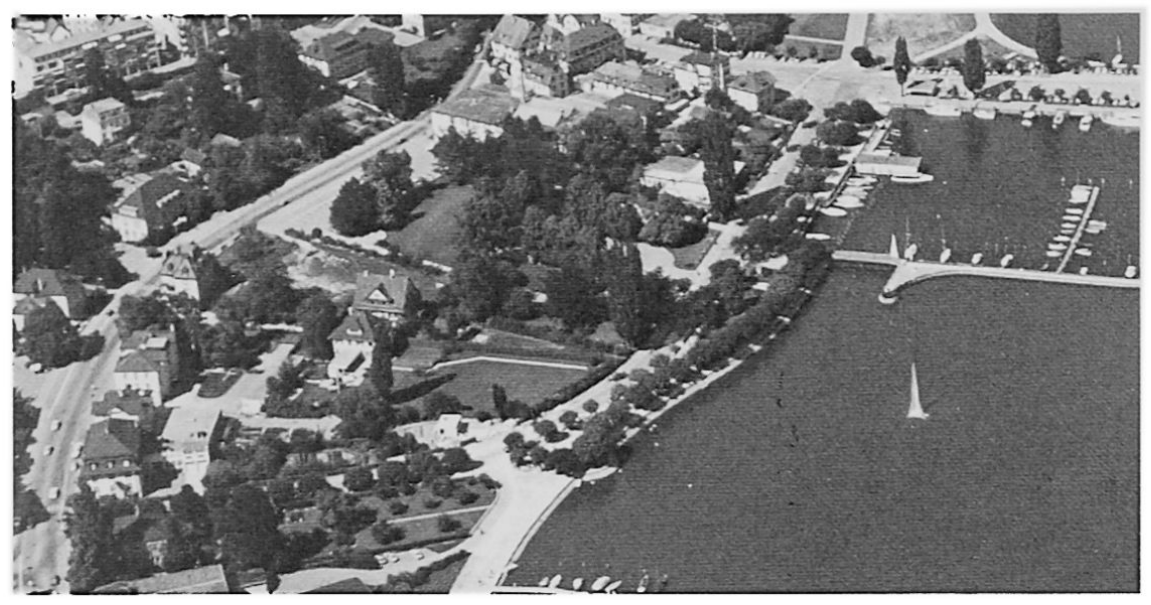

Abb. 6: Sportstyp

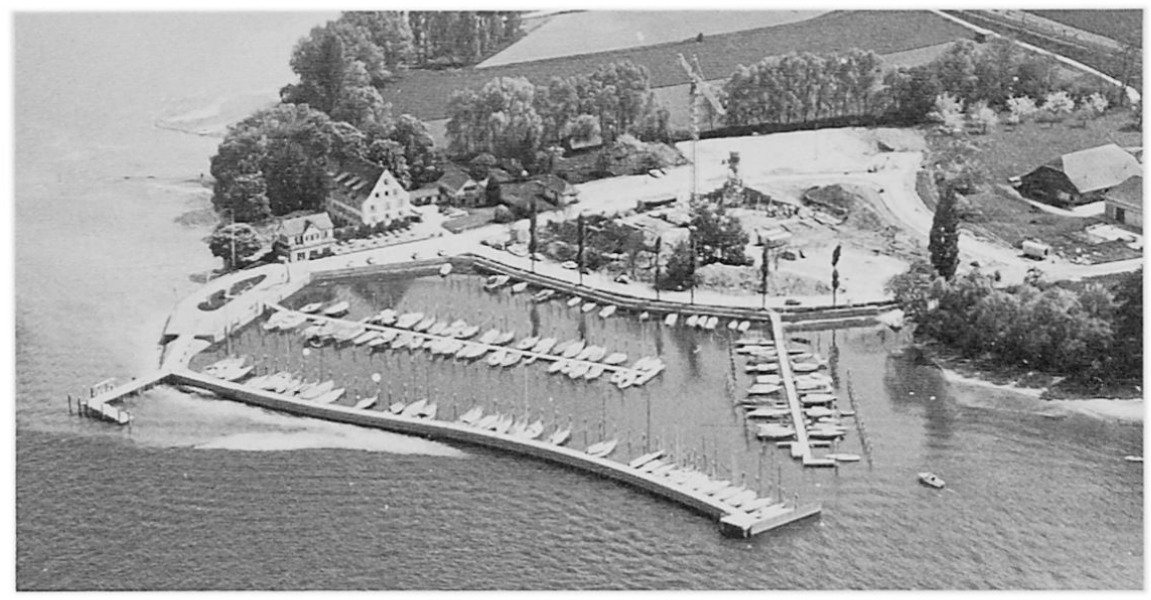

Abb. 7: Bildungs- und Gesundheitstyp

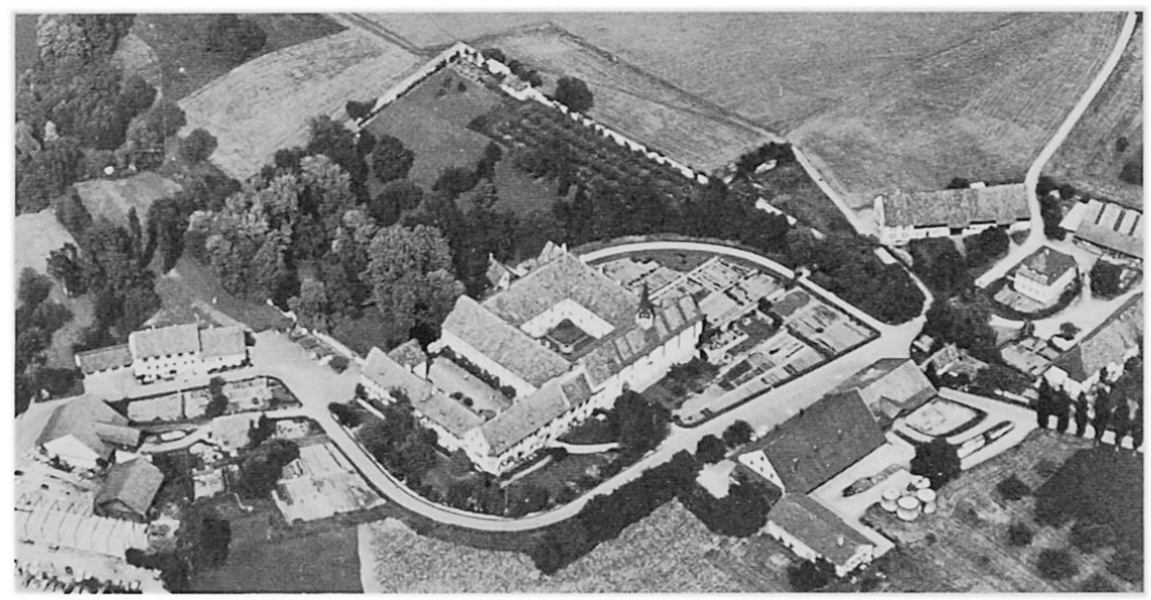

Gewicht innerhalb der Arbeit hatte, soll hier die Arbeitsmethode den Zielen und Maßnahmen vorangestellt werden.

\section{Arbeitsmethode zur Bewertung des landschaftlichen Angebotes für die Erholung5)}

(1) Die meisten Methoden zur Bewertung der Erholungseignung von Landschaften basieren auf einem undifferenzierten Erholungsbegriff. Die Anforderungen der Benutzergruppen an die Landschaft sind jedoch nach unserer Meinung sehr unter- schiedlich. Praxisorientierte Raumplanung und Landschaftsplanung benötigen Grundlagen, um Vorschläge zum Schutz oder zum Ausbau des Angebotes für die Freizeitaktivität einzelner Benutzergruppen ausarbeiten zu können. Die Arbeitsmethode beruht auf einem gruppenspezifischen Ansatz und bewertet Gebiete nach ihrer Eignung für definierte Benutzertypen. Dabei werden das landschaftliche Angebot und die vorhandenen Einrichtungen nach ihrer Eignung für bestimmte Besuchergruppen, hier «Erholungstypen» genannt, bewertet. Den Erholungstypen ordnen wir bestimmte 
Wandertyp

Lagertyp

Promeniertyp

Rundfahrertyp

Sporttyp

Bildungstyp

Gesundheitstyp wandern, spazieren, sich bewegen, Natur beobachten, Ausschau halten, sammeln (Früchte, Pflanzen), rasten, Aussicht genießen, skiwandern;

lagern, picknicken, campieren, zelten, Caravaning, spielen (Rasen- und Ballspiele), ruhen, sitzen, ausspannen, sonnen, Reviere bilden, Reviere ausgestalten (Camping und Wochenendhaus), baden (an frei zugänglichen Ufern);

promenieren, flanieren, einkehren, zuschauen, Leute beobachten, gesehen werden, ausruhen, sitzen;

autofahren, autowandern, besichtigen, einkehren, promenieren, spazieren gehen, rundwandern;

Leichtathletik treiben, trainieren, Ballspiele (Tennis usw.), schwimmen, wasserskifahren, segeln, rudern, bootfahren, golfspielen, reiten;

sich weiterbilden (diskutieren, lesen) besichtigen, kulturelle Veranstaltungen und historische Gebäude besuchen, einkehren.

kuren, baden, Therapie, sonst wie Promeniertyp.
Motivationen und Freizeitaktivitäten sowie aus ihnen abgeleitete Anforderungen an die Landschaft zu. Die Erholungssuchenden und Sporttreibenden haben sehr unterschiedliche und oft gegensätzliche Wünsche und Anforderungen. Es wird eine umfassende Zahl Angebotskriterien hinzugezogen, diese werden aber nur umschrieben oder grafisch dargestellt, auf eine Quantifizierung wird verzichtet.

(2) Jeder Typ stellt andere Anforderungen an das Erholungsgebiet. So meiden die einen zivilisationsnahe Gebiete und stark begangene Wege (Wandertyp), andere sind auf besondere Einrichtungen angewiesen (Rundfahrertyp, Sporttyp) und bevorzugen größere Besucherdichten (Promeniertyp).

(3) Die Typisierung geht von der Voraussetzung aus, daß gewisse Tätigkeiten häufig miteinander gekoppelt auftreten und von bestimmten Gruppen ausgeführt werden. Das bedeutet jedoch nicht, daß bestimmte Erholungstypen auf bestimmte Personengruppen festgelegt werden können oder mit ihnen identisch sind. In der Regel dürfte sich jeder Erholungssuchende in unterschiedlichem Maße und zu verschiedenen Zeiten auch anderen Typen zugehörig fühlen.

(4) Es werden grundsätzlich folgende Typen unterschieden: Wandertyp, Freiraumtyp, Lagertyp, Rundfahrertyp, Promeniertyp, Sporttyp, Bildungstyp und Gesundheitstyp (siehe Tabelle 1 und Abbildungen).

\section{Ziele und Maßnahmen der Seeuferplanung}

Hier können nur einige Ziele und Maßnahmen der Seeuferplanung in Form von Postulaten aufgeführt werden. Ihre Detaillierung ist den entsprechenden Berichten zu entnehmen.
(1) Ziele - Landschaftsschutz

- Schutz der historischen Kulturlandschaft und der wenigen noch naturnahen Gebiete.

- Besonderer Schutz der wenigen unüberbauten und naturnahen Uferzonen vor baulichen Nutzungen.

- Entlastung der Uferzone und Reinhaltung der Gewässer.

(2) Ziele - Erholung

- Der künftig steigenden Belastung ist vorzubeugen.

- Für die fünf sehr unterschiedlichen Teillandschaften des Ufergebietes sind verschiedenartige Erholungskonzepte anzustreben. Sehr vereinfacht und zusammengefaßt:

Zielsetzung Rhein und Untersee; 1. Priorität hat hier die Förderung der natur- und landschaftsbezogenen Erholungsaktivitäten (Aktivitäten des Wander-, Freiraum-, Lagertyps).

Zielsetzung Obersee; 1. Priorität hat hier die Förderung der mehr an Einrichtungen gebundenen Aktivitäten in den Zentren (Aktivitäten des Sport-, Rundfahrer-, Promeniertyps).

- Ausweitung der Erholungseinrichtungen eher landeinwärts als entlang der Ufer und Aufwertung des schönen Hinterlands.

- Öffentlicher Zugang zum Ufer und Waldrand.

- Durchgehender, ufernaher Wanderweg und Uferfreihaltung.

- Bei Erholungseinrichtungen ist dem Landschaftsschutz vermehrt Rechnung zu tragen.

- Sicherung von Flächen für die Erholung der Öffentlichkeit.

- Entlastung durch Konzentration der Erholungseinrichtungen.

- Ausbau bestehender touristischer Zentren. 


\section{Wandertyp}

Faktor notwendig:

- Aussichtspunkte, Aussichtslagen

- mäßiger bis hoher Waldanteil

- Anteil an Naturwiesen

- Anteil an Gebieten extensiver Landwirtschaft

- Schutzgebiete (Natur-, Landschaftsschutz)

- Natur- und Kulturobjekte

- vorhandenes Wanderwegnetz

Faktor aufwertend:

- Naturlandschaft

- Kleingliedrigkeit

- nebelarme Gebiete

- südexponierte Lagen

- mittlere Höhenlagen

- geringe Siedlungsdichte

- Ausblick auf Gewässer, Berge (Fernsicht)

- Geländekulissen

- Gasthöfe, Rastplätze

Faktor störend:

- Autowanderstrecken, dichtes Straßennetz

- hohes Verkehrsaufkommen

- starke und dichte Besiedlung

- andere Immissionen (Fluglärm usw.)

- Gebiete mit hohen Besucherdichten

- Ferienhauszonen
Lagertyp

Faktor notwendig:

- gute Begehbarkeit

- südexponierte, besonnte Lagen

- mild-warmes Klima

- nebelarme Gebiete

- Anteil zugänglicher Gewässer

- Waldanteil

- viel Waldrandeffekt

- Anteil an extensiver Landwirtschaft

- Parkmöglichkeiten

- Flächen zum Lagern und Spielen

- Flächen mit Möglichkeit der Revierbildung

Faktor aufwertend:

- Kulturlandschaft

- attraktive Geländekulisse

- Aussichtslagen

- Rast-, Picknickplätze

- Einkehrmöglichkeiten

- Sport- und Spieleinrichtungen

- gute Erreichbarkeit

Faktor störend:

- zu hoch gelegene Flächen (Klima, Relief)

- sehr flache Gebiete (wenn ohne Gewässer)

- häufiger Nebel

- viel Schnee

- feuchte Gebiete

- dichte Besiedlung

- hohes Verkehrsaufkommen

- schlechte Erreichbarkeit mit privaten und öffentlichen Verkehrsmitteln

\section{Promeniertyp}

Faktor notwendig:

- flaches Gelände

- mildes Klima

- nebelfreie Gebiete

- gute Begehbarkeit

- Promenaden, Parks

- Parkplätze

- Ausflugsziele mit Attraktionen

- Restaurationsbetriebe

- hohe Besucherdichte

- gute Erreichbarkeit für private und öffentliche Verkehrsmittel

Faktor aufwertend:

- Siedlungsnähe

- Randeffekte, Randsituationen (Gewässer, Waldrand)

- Gewässer

- Schutzgebiete

Faktor störend:

- steiles Gelände

- Schnee, Wind, Kälte

- große Waldgebiete

- viel Autoverkehr

- schlechte Zugänglichkeit
(3) Ziele-Tourismus

- Langfristig müssen zusätzliche ergänzende Programme und Angebote für Erholungsaktivitäten zur Saisonverlängerung aufgrund des natürlichen landschaftlichen Potentials verstärkt entwickelt werden bzw. bestehende Erholungsformen erhalten und gefördert werden. Beispiele: Urlaub für ältere Leute/aktiver Urlaub/Familienurlaub/Ferien auf dem Land/Sporturlaub/Wanderurlaub.

\section{(4) Ziele - Camping}

- Restriktionen und vermehrte Auflagen bei Campingplätzen: Standorte für neue Campingplätze dürfen nicht an den für die Erholung attraktiven Situationen und an exponierten Lagen ausgeschieden werden (Fluß-/Seeufer/Hangkanten). Bei den bestehenden Campingplätzen sollten die für die Erholung attraktivsten Gebiete, z. B. die Uferzone, den Erholungssuchenden zugänglich sein (Mindestabstand vom Ufer: $30 \mathrm{~m}$ ). Die für die Erholung attraktivsten Gebiete (Wasserflächen, Ortslagen, Hafenanlagen, Badeplätze, 
Waldgebiete) sollten aber von Campingplätzen aus in Fußgängerdistanz erreichbar sein. Die Campingplätze sollten nicht unmittelbar an Hauptverkehrsstraßen liegen oder müssen zumindest hinreichenden Lärmschutz aufweisen.

(5) Ziele - Wassersport und Schiffahrt

Die meisten der daraus genannten Ziele sind identisch mit der Verordnung der internationalen Schiffahrtskommission über die Schiffahrt auf dem Bodensee, 1976 (z. B. Schutzstreifen 300 m, Langsamfahrzone, PS-Beschränkungen).

Aus den Kapazitätsberechnungen, aber auch aufgrund anderer Unterschiede zwischen Obersee, Untersee und Rhein (wie Ufernutzung, Anteil Schutzgebiete, Wasserverschmutzung) wurden zudem Ziele formuliert.

- Untersee-Rhein:

Hier sollten die Bootszahlen nicht wesentlich erhöht werden.

- Obersee:

Neue Hafenanlagen sollten dort eingerichtet werden, wo die Uferzonen bereits überbaut sind. Die geringen Restflächen mit natürlichem Ufer am Obersee sollten nicht durch neue Hafenanlagen zerstört werden.

(6) Ziele - Hafenanlagen

- Nur wenige, große und gut ausgebaute Anlagen: Es sind eher große Anlagen an wenigen Orten, als kleine an vielen Orten auszubauen (Mindestgröße $=200$ Bootsliegeplätze, Bootszentren, Gemeinschaftsanlagen). Neue Häfen sind nur in Verbindung mit weiteren Einrichtungen auszubauen oder zu erweitern.

\section{Beurteilung der Ergebnisse}

\section{Ergebnisse der Seeuferplanung}

Mit dem Abschluß des Auftrages der Seeuferplanung hat die eigentliche Seeuferplanung auf lokaler, regionaler und kantonaler Ebene eigentlich erst begonnen. Die Umsetzung des ganzen Programmes an Zielen und an konkreten Maßnahmen braucht ungemein Zeit. Einzelne Maßnahmen wurden bisher realisiert. Andere wurden nicht weiterverfolgt. Hierzu einige Gedanken:

\section{(1) Kontinuität/Weiterführung}

Wie eingangs erwähnt, hat die Seeuferplanung den Charakter eines regionalen Richtplanes. Die Seeuferplanung wird von den kommenden kantonalen Teilrichtplänen übernommen und präzisiert werden. Es war von großer Wichtigkeit, daß die Ziele und Maßnahmen wiederholt in den entsprechenden Gremien (Kantonale Verwaltung/Gemeindebehör-
den/Presse) diskutiert und überprüft wurden. Die Gremien der koordinierten Regional- und Kantonalplanung (Regionalplanungsgruppen, Fachkommissionen, Planungsrat und Planungsleitung) sind heute zu Trägern dieser Aufgaben geworden. Einzelne Teilaspekte werden außerdem in Arbeitsgruppen weiterverfolgt, z. B. das Bootshafenkonzept.

\section{(2) Verbindlichkeit}

Erst das kantonale Baugesetz von 1977 schafft eine kantonale Rechtsgrundlage für regionale oder kantonale Teilrichtpläne. Die Seeuferplanung gilt heute als ein Entwurf eines regionalen Richtplanes. Die Aussagen sind für Private und Gemeinden informell und unverbindlich. Die kantonalen Amtsstellen sind gehalten, bei ihren Sachplanungen möglichst den Empfehlungen zu folgen. Trotzdem wurden viele Maßnahmen bisher - sozusagen freiwilligrealisiert. 1976 wurde die internationale Verordnung über die Schiffahrt auf dem Bodensee inkraft gesetzt. Zusätzlich zum kantonalen Baugesetz sind weitere kantonale Gesetze in Vorbereitung, z. B. Natur- und Heimatschutzgesetz und Fremdenverkehrsgesetz. Hiermit wurden geeignete Rechtsinstrumente geschaffen oder werden vorhanden sein, um die Ziele des Natur- und Landschaftsschutzes rechtskräftig zu sichern und die provisorischen Schutzmaßnahmen des Dringlichen Bundesbeschlusses abzulösen.

Da im Kanton Thurgau nach kantonalem Baugesetz der Kanton nur Richtpläne aber keine Nutzungspläne erläßt, d.h. die Nutzungsplanung ausschließlich den Gemeinden vorbehalten bleibt, wird der Nutzungsplanung der Gemeinden nach wie vor große Bedeutung zur Sicherung der Schutzinhalte beizumessen sein.

\section{(3) Konkretisierung}

Im Rahmen der kantonalen und regionalen Richtplanung insbesondere durch die Teilrichtpläne Landschaft und öffentliche Bauten/Anlagen werden viele Ziele und Maßnahmen weiter konkretisiert werden können.

\section{(4) Verwirklichung}

Wichtige Programmpunkte der Seeuferplanung wurden verwirklicht. Hier sollen einige Beispiele genannt werden:

- Das Projekt einer Hochleistungsstraße, der T(N) 13 über den Seerücken zwischen Eschenz und Kreuzlingen wurde aufgegeben. Das Projekt der $\mathrm{N} 4 \mathrm{~b}$ im Bereich des Scharenwaldes/Rheins wurde zumindest langfristig zurückgestellt.

- Einzelne Gemeinden haben ihre Baugebiete in den landschaftlich empfindlichen Lagen reduziert, z. B. Diessenhofen und Steckborn.

- Bisher wurden einige Bootshäfen konzeptgemäß realisiert (Romanshorn, Steinach). Die Bootsta- 
tionierung am Untersee und Rhein wird von der kantonalen Verwaltung äußerst einschränkend behandelt.

- Der Erholungsschwerpunkt Steckborn/Kunstseideareal konnte mit öffentlichen Mitteln von Bund, Kanton und Gemeinde gesichert werden. Hier sind Seeuferpark, Bootshafen, Hallenbad und weitere Erholungsangebote geplant. Die alte schutzwürdige Substanz des Klosters Feldbach und der Gehölzbestand wurden gesichert und werden zu Teilen des geplanten Seeuferparks werden.

- Im Gebiet «Schupfi» (Diessenhofen) konnte die Entwicklung eines Campingplatzes zugunsten der Freihaltung der Landschaft eingeschränkt werden. Der vorgeschlagene Uferwanderweg in diesem Gebiet wird bald verwirklicht werden.

\section{(5) Nichtverwirklichung}

Viele Ziele und Maßnahmen konnten nur teilweise oder noch gar nicht verwirklicht werden. Hier sollen nur drei Problemkreise herausgegriffen werden: Die Sicherung der Schutzgebiete bzw. die Uferfreihaltung, die Bootshafenfrage und die Berücksichtigung der Schutzziele und Schutzmaßnahmen bei anderen öffentlichen Vorhaben.

- Die ufernahen Gebiete sind nach wie vor beliebte Baugebiete, Nachfrage und Baudruck in diesen Gebieten sind groß. Die Gemeinden haben das Interesse, Bauzonen für gute Steuerzahler auszuscheiden und zu erhalten. Der Versuch diese Entwicklung in einigen Gebieten zu bremsen bzw. sogar rückgängig $\mathrm{zu}$ machen, sind oft gescheitert. Die Freihaltung der Uferparzellen als geschützte Ufervegetation entsprechend Bundesgesetz über den Natur- und Heimatschutz, ist nur in einzelnen Fällen möglich. Durch die Abwassersanierung mancher Ufergrundstücke nach $\mathrm{Ge}$ wässerschutzgesetz wird die Verdichtung heute oft erst lückenhaft überbauter Uferparzellen sogar noch beschleunigt. Auch Landschaftsteile, die heute durch den provisorischen Bundesbeschluß als Landschaftsschutzgebiete geschützt sind, sind nach wie vor bedroht. Eine abschlieBende Sicherung dieser Landschaftsteile ist nicht gegeben.

- Die Entwicklung im Bootssport ist groß. Die Bootszahl im Thurgau hat sich zwischen 1970 und 1978 trotz Restriktionen durch Wasserfahrzeugsteuer und internationale Verordnung über die Schiffahrt (1976) fast verdoppelt. Die Zahl der Einzelbojen nimmt zu. Bojenfelder werden nur widerwillig zugunsten von Bootshäfen aufgelöst. Auch im Untersee/Seerheingebiet werden laufend Projekte von neuen Bootshäfen mit zusätzlichen Bootsplätzen erarbeitet.

- Die Schutzmaßnahmen richteten sich bisher vor allem an die Grundeigentümer. Der Staat (Gemeinde, Kanton, Bund) mit seinen Projekten wirkt oft noch stärker landschaftsverändernd als die privaten Grundeigentümer. Die öffentlichen Körperschaften wurden bisher zuwenig auf die Schutzziele verpflichtet. Hochspannungsleitungen durch Landschaftsschutzgebiete, Straßenprojekte entlang oder durch Naturschutzgebiete, Uferverbauungen in naturnahen Uferpartien sind Beispiele dafür, daß die Frühkoordination und die Verpflichtung auf die Schutzziele nicht oder nur sehr oberflächlich erfolgten.

\section{Beurteilung der Arbeitsmethode}

(1) Die angewendete Methode ist als ein Arbeitsinstrument und Hilfsmittel der Planung zu verstehen. Mithilfe der Eignungsbewertung konnten Ziele und Maßnahmen auf ein Konzept ausgerichtet werden. Die Arbeitsmethode ist ein wichtiger Planungsschritt zu Beginn der Arbeiten. Bei der Verwirklichung der Ziele und Maßnahmen dem eigentlichen Schwerpunkt der Planung bringt die Arbeitsmethode selbstverständlich wenig.

(2) Empirisches Material über das Freizeitverhalten fehlt allgemein, dies gilt besonders für die Schweiz. Ganz besonders aber gilt es, wenn man Erholungsplanungen kleinräumig für bestimmte Regionen/Gemeinden kurzfristig durchführen muß. Oft ist man gezwungen, eigene Zählungen und Beobachtungen durchzuführen. Bei der Arbeit über das Thurgauer Rhein- und Bodenseeufer wurden an bestimmten Tagen Befliegungen durchgeführt und die dabei gemachten Luftaufnahmen wurden anschließend ausgewertet (laienhaft!).

(3) Die Arbeitsmethode läßt sich nicht von Gebiet zu Gebiet mit dem gleichen Ansatz durchführen. Landschaftliches Angebot und Einrichtungen, die Erwartungen der Einwohner und Besucher, wie auch die Erreichbarkeit sind von Gebiet zu Gebiet unterschiedlich. Der Ansatz mußte bei anderen Arbeiten, z. B. im Kanton Schaffhausen und im Kanton Zug jeweils neu überdacht werden. Entscheidend scheint aber zu sein, daß der Ansatz für den Außenstehenden anschaulich und nachvollziehbar bleibt (in der Bewertung/Konzept und den Maßnahmen).

(4) Über die Belastbarkeit der Landschaft durch einzelne Erholungsformen weiß man heute noch sehr wenig. Darum sollten Vorschläge für neue Einrichtungen am ehesten dort vorgesehen werden, wo sie dringend nötig sind bzw. dort, wo schon zerstörte Landschaftsteile mit neuen Einrichtungen «saniert» werden können. 
(5) Die Arbeitsmethode stellt hohe Anforderungen an den Bearbeiter. Gute Gebiets- und Besucherkenntnisse sind Voraussetzung. Sie ist damit zeitaufwendig. Der Bearbeiter schafft sich aber mit der Bewertung die Grundlagen für Konzepte, Maßnahmen und deren Umsetzung in die Realität. Die Methode - dies zeigen die gegenwärtigen Arbeiten des kantonalen Landschaftsrichtplanes - lässt sich gut kombinieren und überlagern mit der Landschaftsbewertung der weiteren landschaftlichen Qualitäten des Landschaftsschutzes (biologische, hydrologische, geologische, ökologische, kulturgeschichtliche, ästhetische).

1) C. FINGERHUTH/M. SCHWARZE/R. WICKL: Erholungs- und Landschaftsschutzplanung thurgauisches Bodensee- und Rheinufer, Selbstverlag, Zürich, 1972, (Gesamtbericht $=2$ Arbeitsberichte), 284 Seiten.

2) dito; überarbeiteter Auszug, Zürich 1975, 98 Seiten.

3) Planungsleitung der Regional- und Kantonalplanung Thurgau, Leitbild Entwurf, Frauenfeld, Juni 1978, 61 Seiten.

4) dito; Seiten $42 / 46 / 47$.

5) C. FINGERHUTH/S. HESSE/H. KNOPS/M. SCHWARZE: Arbeitsmethode zur Bewertung der Erholungseignung eines landschaftlichen Angebotes für verschiedene Typen von Erholungssuchenden, Selbstverlag, Zürich, 1972, 35 Seiten.

\section{Résumé}

L'aménagement des rives thurgoviennes du lac de Constance et du Rhin a été demandé en 1970. Ce mandats d'étude est terminé en 1974. Les objectifs de ces travaux et les dispositions qu'ils préconisent sont toujours valables et se poursuivent dans le cadre de l'aménagement régional et cantonal du territoire (Projet de conception directrice cantonale 1978 , projets de plans directeurs sectoriels, en particulier plan directeur sectoriel du paysage). La planification des rives du lac n'est donc pas terminée, elle est en constante évolution.

On peut considérer le plan de 1974 pour les rives du lac comme projet d'un plan directeur régional du paysage. Les informations qu'il fournit, ses objectifs et ses dispositions sont inofficiels, donc n'engagent personne. Cependant, on ne saurait sousestimer l'effet produit par ces informations. Bien des choses ont été réalisées depuis, les mesures de promotion ayant bien ententu été plus facilement acceptées que les restrictions. Pour réaliser lesdits objectifs et lesdites dipositions, plusieurs instruments juridiques ont été créés. D'autres sont envisagés.

Afin d'évaluer l'offre de paysages récréatifs, on a conçu et utilisé pour la planification des rives du lac une méthode de travail particulière, dite des «genres de récréation». Cette méthode a largement aidé à définir les objectifs et les mesures à prendre. 